\title{
Robust speed controller design for permanent magnet synchronous motor based on gain-scheduled control method via LMI approach
}

\author{
Mohammad Hossein Mousavi $^{1}$ (D) $\cdot$ Mohammad Ehsan Karami ${ }^{2} \cdot$ Mojtaba Ahmadi ${ }^{1} \cdot$ Peyman Sharafi $^{1} \cdot$ Farhad Veysi $^{1}$
}

Received: 21 February 2020 / Accepted: 27 August 2020 / Published online: 14 September 2020

(c) Springer Nature Switzerland AG 2020

\begin{abstract}
In recent years, permanent magnet synchronous motors (PMSMs) have received more attention in industries due to their higher efficiency in comparison to induction motors. Moreover, they play a growingly important role in applications where variable speeds are necessary. This paper is concerned with the speed control of PMSMs, and the controller design procedure is developed in two steps. Firstly, a novel robust controller is designed via linear matrix inequalities (LMIs) approach in order to guarantee the robustness of the speed control under the load variation and uncertainty. The design of the main LMI, uncertainty, and disturbance elimination LMI involves several steps which are presented in the paper. For comparison, classic state feedback controller and proportional-integral-derivative controller with parameters optimized by genetic algorithm are implemented in different scenarios. Secondly, a gain-scheduled controller is designed and simulated. The controller design conditions are derived in terms of LMls, which can be solved via convex optimization in MATLAB using YALMIP toolbox. It is observed that instead of using one robust controller for all operating points, several ones can be used, and by measuring the speed feedbacks, the control system opt for the appropriate controller. The suggested gain-scheduled control method via LMI approach gives excellent performance over the complete operational range, and the results validate the robustness and effectiveness of the proposed method.
\end{abstract}

Keywords Permanent magnet synchronous motor · Robust control · Gain-scheduled control · Linear matrix inequality

\section{Introduction}

In the last decades, permanent magnet synchronous motors (PMSMs) have been used in a wide variety of applications, including electrical vehicles, robotics, aerospace and different kinds of industries. Simple structure, low volume, low electrical losses, and high efficiency are the advantages of PMSMs over other electric motors. Although PMSMs have recently gained immense popularity in electrical systems, there are still some weaknesses that the most important one is the complicated speed control method due its nonlinear characteristics. Thus, speed control of these motors has always been of interest to scientists and engineers [1-4].
Many researches have been conducted to identify the best PMSM speed control method in the last years. Nonlinear control [5-8], adaptive control $[9,10]$ and robust control $[11,12]$, compared to other methods have been more popular due to their efficient performance. Of course, some other control methods are also worth mentioning such as sliding-mode control (SMC) [6, 13-15], neurofuzzy control (NFC) [16-18], and generalized predictive and sliding-mode control (GPSMC) [19, 20].

Robust control is one of the most common strategies to design control systems that emphasizes on the stability and robustness under parameter variations and uncertainties. The design purpose is to create a control system in which system changes have the least effect on output. In

Mohammad Hossein Mousavi, mh.mousavii13@gmail.com | 'Department of Electrical Engineering, Faculty of Engineering, Razi University, Kermanshah, Iran. ${ }^{2}$ Department of Electrical Engineering, Faculty of Engineering, University of Guilan, Rasht, Iran. 
other words, increasing the reliability of the system is the most important goal of the robust control design [21, 22].

A high volume of studies in robust control implementation clearly shows the appropriate performance of this controller under different conditions. Of course, robustness is not an absolute concept and any controller structure is somewhat robust and hence, many known control methods, such as proportional-integral-derivative (PID) control or SMC, have some sense of robustness [23-25]. Indeed, [25] has proposed an integral SMC algorithm based on field-oriented control (FOC) with space vector pulse width modulation (SVPWM) for PMSM speed regulation system. It was proved that under the load variation, SMS speed control system had better robustness than PI controller. In [26], also a two-degree-of-freedom controller including two internal electric current loops driven by proportional and PI controllers has been presented. The results validated the global exponential stability and robustness with respect to uncertainties. Additionally, to guarantee the robustness and fast dynamic response of the PMSM, an adaptive speed control scheme based on recurrent Elman neural network (RENN) in the presence of system disturbances has been considered [27].

For a kind of electric motor like PMSM, many factors can be considered as system uncertainties. These factors may have detrimental effects on the motor leading to failures. Load disturbances, mechanical parameter variation, and unmodeled dynamics are the most significant uncertainties that PMSMs are dealing with $[27,28]$. To accurately control the speed of PMSMs in regard to structured and unstructured uncertainties, robust controllers are employed to compensate the negative effects of the perturbations. It is to be noted that the robust controllers should be able to control PMSMs at acceptable levels with minimum errors despite mentioned troubles and it is also essential to achieve strong robust performance and fast response for PMSMs [20].

As it could be seen from the last related studies, classic controllers, proportional controllers, integral controllers and controllers based on the super-recursive algorithms in the permanent magnetic synchronous motor control system do not have a remarkable performance in tracking the rotor speed as well as rejecting disturbances and noises. In this study, it has been tried to cover and eliminate these defects, so a robust controller based on gain-scheduling method is suggested. The linearization technique is successfully applied to electrical systems. Usually, each linear model is based on a fixed operating point. When the operating point changes, the initial model will no longer be functional due to the nonlinear nature of the system.
Because of the extensive changes in the dynamic characteristics of electrical systems, the gain-scheduling method is a suitable design strategy to strengthen the robustness of the system. Gain-scheduling control can demonstrate a good performance in dealing with these vexingly complicated issues compared to previous controllers. More effective speed tracking, significant noise reduction and faster response are the main advantages of this technique.

Therefore, given the aforementioned problems, this paper, proposes a specific robust controller based on gainscheduled method via LMI approach to ensure robustness and fast dynamic response. First, a robust controller via uncertainties elimination LMIs is designed. Rotor inductance and moment of inertia are system uncertainties, and the load torque variation is the applied disturbance. To verify the performance and robustness of this controller, the classic state feedback controller as well as the PID controller [with parameters optimized by genetic algorithm $(\mathrm{GA})]$ are designed and compared with the robust one. The performance and reliability of the designed robust controller can be seen to be higher. Next, the robust performance improvement is addressed using the gainscheduled method. Due to the different reference speeds tested and disturbances applied to the motor such as load torque changes, boundaries are considered for the motor output speed variations and therefore an interval is formed. The interval itself is divided into several smaller intervals and a robust controller is designed for each interval to switch between multiple robust controllers instead of using one, and actually gain-scheduled control is implemented. Finally, in order to prevent sudden gain changes and reduce the effects of disturbances, the gains are mildly modified based on a defined function. The simulation results show that the proposed control method is reliable and robustly effective for the speed control of the PMSM over a wide range of operations.

This paper is organized as follows. In Sect. 2, the mathematical model of a PMSM system is introduced. In Sect. 3, preliminaries and the design steps of robust speed controller using LMI approach is represented. Section 4 presents the simulations and the results of proposed methods using MATLAB. Conclusions are summarized in Sect. 5 .

\section{Mathematical model of PMSM}

The mathematical model of a surface-mounted PMSM in rotor fixed frame ( $d-q$ frame) is written in the following form $[28,29]$ : 


$$
\begin{array}{r}
\dot{i}_{d}=-\frac{R_{\mathrm{s}} i_{d}}{L_{d}}+p \omega i_{q}+\frac{u_{d}}{L_{d}} \\
\dot{i}_{q}=-\frac{R_{\mathrm{s}} i_{q}}{L_{q}}-p \omega i_{d}-\frac{p \omega \phi_{\mathrm{m}}}{L_{q}}+\frac{u_{q}}{L_{q}} \\
\dot{\omega}=\frac{K_{\mathrm{t}} i_{q}}{J}-\frac{B \omega}{J}-\frac{T_{\mathrm{L}}}{J} \\
\dot{\theta}=\omega
\end{array}
$$

$$
\begin{aligned}
& A=\left[\begin{array}{cccc}
-\frac{R}{L} & 0 & -\frac{p \phi_{\mathrm{m}}}{L} & 0 \\
0 & 0 & 1 & 0 \\
-\frac{K_{\mathrm{t}}}{J} & 0 & -\frac{B}{J} & 0 \\
0 & -1 & 0 & 0
\end{array}\right], B=\left[\begin{array}{llll}
\frac{1}{L} & 0 & 0 & 0
\end{array}\right]^{\top} \\
& D_{\mathrm{w}}=\left[\begin{array}{cc}
0 & 0 \\
0 & 0 \\
-\frac{1}{J} & 0 \\
0 & 1
\end{array}\right], C_{\mathrm{z}}=\left[\begin{array}{llll}
0 & 0 & 0 & 1
\end{array}\right]
\end{aligned}
$$

\section{Controller design}

In this section, the goal is to design a robust controller by obtaining an uncertainty and disturbance elimination LMI.

Consider the following system, including disturbance and uncertainty

where $L_{q}$ and $L_{d}$ are the stator winding inductance of $d-q$ axes which $L_{d}=L_{q}=L, u_{d}, u_{q}, i_{d}$ and $i_{q}$ are the stator voltages and currents of $d-q$ axes, $R_{\mathrm{s}}$ is the stator resistance, $\omega$ is the rotor velocity, $\theta$ is the rotor angle position, $p$ is the number of pole pairs, $K_{\mathrm{t}}=\frac{3}{2} p \phi_{\mathrm{m}}$ is the torque constant, $\phi_{\mathrm{m}}$ is the magnetic flux of rotor, $T_{\mathrm{L}}$ is the load torque, $J$ is the moment of inertia and $B$ is the viscous friction coefficient.

The state equation of proposed system can be represented in the following form:

$\left\{\begin{array}{r}x(\dot{t})=A x(t)+B u(t)+D_{\mathrm{w}} \mathrm{d}(t) \\ z(t)=C x(t)+D \mathrm{~d}(t)\end{array}\right.$

whereu $(t)$ and $\mathrm{d}(t)=\left[\begin{array}{ll}\bar{\theta} & T_{\mathrm{L}}\end{array}\right]^{\top}$ are the control input and disturbance input. $\bar{\theta}$ is the desired angular position, $D_{\mathrm{w}}$ is the disturbance matrix and $D=0$. Also, $z(t)$ refers to the performance output and $C$ is equivalent to $C_{z}$ which all its parameters are set to be zero except $e(t)$. This is due to error minimization. $e(t)$ is a defined auxiliary variable for tracking error with the formulation as follows.

$e(\dot{t})=\bar{\theta}-\theta(t)$

The reference current $i_{d}$ is usually assumed to be zero to eliminate the influence between $\omega, i_{d}$ and $i_{q}$ [30]. Therefore $u(t)=u_{q}(t)$.

From (1) and (2) with some changes, $x(t)$ vector can be obtained as

$x(t)=\left[i_{q}(t) \theta(t) \omega(t) e(t)\right]^{\top}$
$\left\{\begin{array}{l}\dot{x}=A(t) x+D(t) w+B(t) u \\ z=C_{1}(t) x+D_{11}(t) w+D_{12}(t) u \\ y=C_{2}(t) x+D_{21}(t) w+D_{22}(t) u\end{array}\right.$

where

$$
\left\{\begin{array}{l}
A(t)=A+\Delta A(t) \\
B(t)=B+\Delta B(t) \\
D(t)=D+\Delta D(t) \\
C_{1}(t)=C_{1}+\Delta C_{1}(t) \\
D_{12}(t)=D_{12}+\Delta D_{12}(t)
\end{array}\right.
$$

$$
\left\{\begin{array}{l}
\Delta A(t)=M_{A} F(t) N_{A} \\
\Delta B(t)=M_{B} F(t) N_{B} \\
\Delta D(t)=M_{D} F(t) N_{D} \\
\Delta C_{1}(t)=M_{C_{1}} F(t) N_{C_{1}} \\
\Delta D_{12}(t)=M_{D_{12}} F(t) N_{D_{12}}
\end{array}\right.
$$

and, $u$ is control input, $w$ is disturbance/reference input, $y$ is sensed output and $z$ refers to performance output.

From structure bounded uncertainty definition,

$\Delta A(t)=A_{1}(t) A_{2}(t) A_{3}(t)$.

where $A_{1}(t)=M_{A} \in R^{n \times k}$ and $A_{3}(t)=N_{A} \in R^{l \times n}$ are known real constant matrices an $A_{2}(t)=F(t) \in R^{k \times l}$ which $F(t)$ is the unknown Lebesque-measurable matrix-valued function subject to the following condition [31, 32]:

$F^{\top}(t)=F(t) \leq I \quad \forall t$.

It depends massively on the system sensors whether $x, w$ and $u$ appear in $y$ or not. Here, it is considered that only states are measured: 
$y=x$.

it is also assumed that $D_{11}=D_{21}=D_{22}=0$ and $C_{2}=I$.

In order to design the robust controller using system states,

$u=K x$,

the following conditions need to be satisfied:

(1) The closed-loop system must be asymptotically stable without any disturbance.

(2) Considering disturbance [33]:

$\frac{\|z\|_{I_{2}}}{\|w\|_{I_{2}}} \leq \gamma$

where $\gamma$ is a positive constant $(\gamma>0)$.

$$
\begin{gathered}
j \leq \int_{0}^{\infty}\left(z^{\top} z-\gamma^{2} w^{\top} w\right) \mathrm{d} t+[V(x(\infty))-V(x(0))] \\
=\int_{0}^{\infty}\left(z^{\top} z-\gamma^{2} w^{\top} w+\dot{V}(x)\right) \mathrm{d} t<0
\end{gathered}
$$

If the above term is negative, its integration is negative too, therefore:

$z^{\top} z-\gamma^{2} w^{\top} w+\dot{V}(x)<0$

By embedding $z$ from (7) in the above equation, $\psi_{1}(t)$ matrix is obtained. A new parameter named $\eta=\left[\begin{array}{c}x \\ w\end{array}\right]$ is defined, then

$\left[x^{\top} w^{\top}\right] \psi_{1}(t)\left[\begin{array}{c}x \\ w\end{array}\right]<0$

$\psi_{1}(t)=\left[\begin{array}{cc}{[A(t)+B(t) K]^{\top} P+P[A(t)+B(t) K]+\left[C_{1}(t)+D_{12}(t) K\right]^{\top}\left[C_{1}(t)+D_{12}(t) K\right]} & P D(t) \\ D^{\top}(t) P & -\gamma^{2} l\end{array}\right]<0$

From Lyapunov theory, a system is asymptotically stable when

$V(x)=x^{\top} P x>0$ for $x \neq 0$

$P>0$

$\dot{V}(x)<0$ for $x \neq 0$

where $V(x)$ is Lyapunov candidate function and $P$ is a positive symmetric matrix.

For (1):

$\dot{V}(x)=\dot{x}^{\top} P x+x^{\top} P \dot{x}=x^{\top}[A(t)+B(t) K]^{\top} P x+x^{\top} P[A(t)+B(t) K] x<0$

For (2):

$\dot{V}(x)=x^{\top}[A(t)+B(t) K]^{\top} P x+w^{\top} D(t)^{\top} P x+x^{\top} P[A(t)+B(t) K] x+x^{\top} P D(t) w<0$

If (2) is proved, (1) will also be proved.

$\frac{\|z\|_{1_{2}}}{\|w\|_{I_{2}}}=\sqrt{\frac{\int_{0}^{\infty}\left(z^{\top} z\right) \mathrm{d} t}{\int_{0}^{\infty}\left(w^{\top} w\right) \mathrm{d} t}} \leq \gamma \rightarrow \int_{0}^{\infty} z^{\top} z \mathrm{~d} t \leq \gamma^{2} \int_{0}^{\infty} w^{\top} w \mathrm{~d} t$

Defining a new parameter $j$ which is expressed as below

$j=\int_{0}^{\infty}\left(z^{\top} z-\gamma^{2} w^{\top} w\right) \mathrm{d} t \leq 0$
Lemma 1 (Schur Complement) Given constant matrices $\Omega_{1}, \Omega_{2}, \Omega_{3}$ where $\Omega_{1}=\Omega_{1}^{\top}$ and $\Omega_{3}>0$, then $\Omega_{1}+\Omega_{2} \Omega_{3}^{-1} \Omega_{2}^{\top}<0$ if only if

$\left[\begin{array}{cc}\Omega_{1} & \Omega_{2}^{\top} \\ \Omega_{2} & -\Omega_{3}\end{array}\right]<0$

Lemma 2 Let $D, S$ and $F$ be real matrices of appropriate dimensions with $F$ satisfying $F^{\top} F \leq I$. Then, for any scalar $\varepsilon>0$.

$D F S+(D F S)^{\top} \leq \varepsilon S^{\top} S+\varepsilon^{-1} D D^{\top}$.

Due to the term $\left[C_{1}(t)+D_{12}(t) K\right]^{\top}\left[C_{1}(t)+D_{12}(t) K\right], \psi_{1}(t)$ is a bilinear matrix inequality (BMI).

In order to convert this term to LMI, Lemma 1 (Schur Complement) is used as follows [31, 32].

$$
\begin{aligned}
& \psi_{1}(t)=\left[\begin{array}{rr}
{[A(t)+B(t) K]^{\top} P+P[A(t)+B(t) K]} & P D(t) \\
D^{\top}(t) P & -\gamma^{2} I
\end{array}\right] \\
&+\left[\begin{array}{ll}
C_{1}(t)+D_{12}(t) K \\
0
\end{array}\right]^{\top} I\left[C_{1}(t)+D_{12}(t) K\right.0]<0
\end{aligned}
$$

Therefore, $\psi_{2}(t)$ is formed which can be another representation for above matrix. Using Lemma 1 (See Ref. [34] which proves the functionality of this Lemma): 


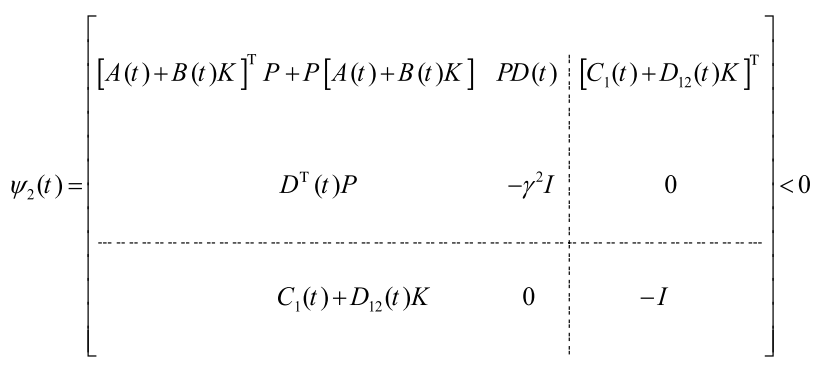

Assume $\left\{\begin{array}{l}\psi<0 \\ P>0\end{array}\right.$ then, $P^{\top} \psi P<0$.

Also consider the following equations:

$$
\begin{aligned}
& X=P^{-1} \\
& Y=K P^{-1}
\end{aligned}
$$

By using (26) and (27), $\psi_{2}(t)$ can be rewritten as $\psi_{3}(t)$.

$\psi_{3}(t)=\left[\begin{array}{ccc}P^{-1} & 0 & 0 \\ 0 & l & 0 \\ 0 & 0 & l\end{array}\right] \psi_{2}(t)\left[\begin{array}{ccc}P^{-1} & 0 & 0 \\ 0 & l & 0 \\ 0 & 0 & l\end{array}\right]$

It is clear that $\psi_{2}(t)<0$ is equivalent to $\psi_{3}(t)<0$.
$\psi_{3}(t)=\left[\begin{array}{ccc}A(t) X+X A^{\top}(t)+B(t) Y+Y^{\top} B^{\top}(t) & D(t) & X C_{1}^{\top}(t)+Y^{\top} D_{12}^{\top}(t) \\ D^{\top}(t) & -\gamma^{2} I & 0 \\ C_{1}(t) X+D_{12}(t) Y & 0 & -l\end{array}\right]<0$

$\psi_{3}(t)=\psi_{3}+\Delta \psi_{3}(t)$

$\psi_{3}=\left[\begin{array}{ccc}A X+X A^{\top}+B Y+Y^{\top} B^{\top} & D & X C_{1}^{\top}+Y^{\top} D_{12}^{\top} \\ D^{\top} & -\gamma^{2} I & 0 \\ C_{1} X+D_{12} Y & 0 & l\end{array}\right]$

$\Delta \psi_{3}(t)=\left[\begin{array}{ccc}\Delta A(t) X+X \Delta A^{\top}(t)+\Delta B(t) Y+Y^{\top} \Delta B^{\top}(t) & \Delta D(t) & X \Delta C_{1}^{\top}(t)+Y^{\top} \Delta D_{12}^{\top}(t) \\ \Delta D^{\top}(t) & 0 & 0 \\ \Delta C_{1}(t) X+\Delta D_{12}(t) Y & 0 & 0\end{array}\right]$

Substituting (9), (10) and (11) into (32), then (32) can be represented as

$\Delta \psi_{3}(t)=\left[\begin{array}{ccc}\Theta & M_{D} F(t) N_{D} & X N_{C_{1}} F(t) M_{C_{1}}+Y^{\top} N_{D_{12}}^{\top} F(t) M_{D_{12}}^{\top} \\ N_{D}^{\top} F(t) M_{D}^{\top} & 0 & 0 \\ \Gamma & 0 & 0\end{array}\right]$

$\Theta=M_{A} F(t) N_{A} X+X^{\top} N_{A}^{\top} F(t) M_{A}^{\top}+M_{B} F(t) N_{B} Y+Y^{\top} N_{B}^{\top} F(t) M_{B}^{\top}$

$\Gamma=M_{C_{1}} F(t) N_{C_{1}} X+M_{D_{12}} F(t) N_{D_{12}} Y$

(33) can be rewritten as

$$
\begin{aligned}
\Delta \psi_{3}(t)=\left[\begin{array}{r}
M_{A} \\
0 \\
0
\end{array}\right] F(t)\left[\begin{array}{lll}
N_{A} X & 0 & 0
\end{array}\right]+\left[\begin{array}{r}
X N_{A}^{\top} \\
0 \\
0
\end{array}\right] F(t)\left[\begin{array}{lll}
M_{A}^{\top} & 0 & 0
\end{array}\right]+\left[\begin{array}{r}
M_{B} \\
0 \\
0
\end{array}\right] F(t)\left[\begin{array}{lll}
N_{B} Y & 0 & 0
\end{array}\right] \\
+\left[\begin{array}{r}
Y^{\top} N_{B}^{\top} \\
0 \\
0
\end{array}\right] F(t)\left[\begin{array}{lll}
M_{B}^{\top} & 0 & 0
\end{array}\right]+\left[\begin{array}{r}
M_{D} \\
0 \\
0
\end{array}\right] F(t)\left[\begin{array}{lll}
0 & N_{D} & 0
\end{array}\right] \\
+\left[\begin{array}{r}
0 \\
N_{D}^{\top} \\
0
\end{array}\right] F(t)\left[\begin{array}{lll}
M_{D}^{\top} & 0 & 0
\end{array}\right]+\left[\begin{array}{r}
0 \\
0 \\
M_{C_{1}}
\end{array}\right] F(t)\left[\begin{array}{lll}
N_{C_{1}} X & 0 & 0
\end{array}\right] \\
+\left[\begin{array}{r}
X N_{C_{1}}^{\top} \\
0 \\
0
\end{array}\right] F(t)\left[\begin{array}{lll}
0 & 0 & M_{C_{1}}^{\top}
\end{array}\right]+\left[\begin{array}{r}
0 \\
0 \\
M_{D_{12}}
\end{array}\right] F(t)\left[\begin{array}{llll}
N_{D_{12}} & 0 & 0
\end{array}\right]+\left[\begin{array}{r}
Y^{\top} N_{D_{12}}^{\top} \\
0 \\
0
\end{array}\right] F(t)\left[\begin{array}{lll}
0 & 0 & M_{D_{12}}^{\top}
\end{array}\right]
\end{aligned}
$$


By Lemma 2, (36) can be expressed as

$$
\begin{aligned}
& \Delta \psi_{3} \leq \varepsilon_{1}\left[\begin{array}{r}
M_{A} \\
0 \\
0
\end{array}\right]\left[\begin{array}{lll}
M_{A}^{\top} & 0 & 0
\end{array}\right]+\varepsilon_{1}^{-1}\left[\begin{array}{r}
X N_{A}^{\top} \\
0 \\
0
\end{array}\right]\left[\begin{array}{lll}
N_{A} X & 0 & 0
\end{array}\right]+\varepsilon_{2}\left[\begin{array}{r}
M_{B} \\
0 \\
0
\end{array}\right]\left[\begin{array}{lll}
M_{B}^{\top} & 0 & 0
\end{array}\right] \\
& +\varepsilon_{2}^{-1}\left[\begin{array}{r}
Y^{\top} N_{B}^{\top} \\
0 \\
0
\end{array}\right]\left[\begin{array}{lll}
N_{B} Y & 0 & 0
\end{array}\right]+\varepsilon_{3}\left[\begin{array}{r}
a \\
N_{D}^{\top} \\
a
\end{array}\right]\left[\begin{array}{lll}
0 & N_{D} & 0
\end{array}\right]+\varepsilon_{3}^{-1}\left[\begin{array}{r}
M_{D} \\
0 \\
0
\end{array}\right]\left[\begin{array}{lll}
M_{D}^{\top} & 0 & 0
\end{array}\right] \\
& +\varepsilon_{4}\left[\begin{array}{r}
0 \\
0 \\
M_{C_{1}}
\end{array}\right]\left[\begin{array}{lll}
0 & 0 & M_{C_{1}}^{\top}
\end{array}\right]+\varepsilon_{4}^{-1}\left[\begin{array}{r}
X N_{C_{1}}^{\top} \\
0 \\
0
\end{array}\right]\left[\begin{array}{lll}
N_{C_{1}} X & 0 & 0
\end{array}\right] \\
& +\varepsilon_{5}\left[\begin{array}{r}
0 \\
0 \\
M_{D_{12}}
\end{array}\right]\left[\begin{array}{lll}
0 & 0 & M_{D_{12}}^{\top}
\end{array}\right]+\varepsilon_{5}^{-1}\left[\begin{array}{r}
Y^{\top} N_{D_{12}}^{\top} \\
0 \\
0
\end{array}\right]\left[\begin{array}{llll}
N_{D_{12}} Y & 0 & 0
\end{array}\right]=\overline{\Delta \psi_{3}}
\end{aligned}
$$

$$
\begin{aligned}
& \psi_{3}(t)=\psi_{3}+\Delta \psi_{3}(t) \leq \psi_{3}+\overline{\Delta \psi_{3}}<0 \\
& \psi_{4}=\psi_{3}+\overline{\Delta \psi_{3}} \\
& \psi_{3}(t)<0 \rightarrow \psi_{4}<0
\end{aligned}
$$

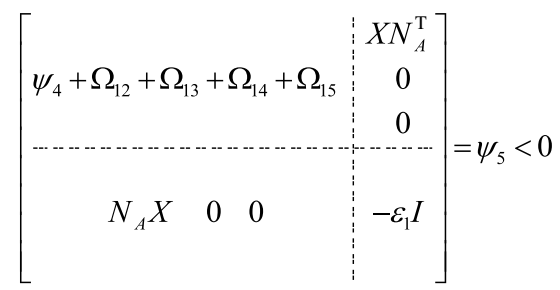

$\Omega_{11}=\varepsilon_{1}^{-1}\left[\begin{array}{r}X N_{A}^{\top} \\ 0 \\ 0\end{array}\right]\left[\begin{array}{lll}N_{A} X & 0 & 0\end{array}\right], \Omega_{12}=\varepsilon_{2}^{-1}\left[\begin{array}{r}Y^{\top} N_{B}^{\top} \\ 0 \\ 0\end{array}\right]\left[\begin{array}{lll}N_{B} Y & 0 & 0\end{array}\right]$

$\Omega_{13}=\varepsilon_{3}^{-1}\left[\begin{array}{r}M_{D} \\ 0 \\ 0\end{array}\right]\left[\begin{array}{lll}M_{D}^{\top} & 0 & 0\end{array}\right], \Omega_{14}=\varepsilon_{4}^{-1}\left[\begin{array}{r}X N_{C_{1}}^{\top} \\ 0 \\ 0\end{array}\right]\left[\begin{array}{lll}N_{C_{1}} X & 0 & 0\end{array}\right], \Omega_{15}=\varepsilon_{5}^{-1}\left[\begin{array}{r}Y^{\top} N_{D_{12}}^{\top} \\ 0 \\ 0\end{array}\right]\left[\begin{array}{lll}N_{D_{12}} & 0 & 0\end{array}\right]$

$\psi_{5}=\psi_{4}+\Omega_{11}+\Omega_{12}+\Omega_{13}+\Omega_{14}+\Omega_{15}<0$

$\psi_{4}+\Omega_{12}+\Omega_{13}+\Omega_{14}+\Omega_{15}+\left[\begin{array}{r}X N_{A}^{\top} \\ 0 \\ 0\end{array}\right] \varepsilon_{1}^{-1}\left[\begin{array}{lll}N_{A} X & 0 & 0\end{array}\right]<0$
Similarly, other $\Omega_{\mathrm{S}}$ are converted into LMIs. Finally, $\psi_{10}$ is presented as follows.

$\psi_{10}=\left[\begin{array}{cccccccc}\Pi & D & X C_{1}^{\top}+Y^{\top} D_{12}^{\top} & X N_{A}^{\top} & Y^{\top} N_{B}^{\top} & M_{D} & X N_{C_{1}}^{\top} & Y^{\top} N_{D_{12}}^{\top} \\ D^{\top} & \varepsilon_{3} N_{D}^{\top} N_{D}-\gamma^{2} I & 0 & 0 & 0 & 0 & 0 & 0 \\ C_{1} X+D_{12} Y & 0 & -I & 0 & 0 & 0 & 0 & 0 \\ N_{A} X & 0 & 0 & -\varepsilon_{1} I & 0 & 0 & 0 & 0 \\ N_{B} Y & 0 & 0 & 0 & -\varepsilon_{2} I & 0 & 0 & 0 \\ M_{D}^{\top} & 0 & 0 & 0 & 0 & -\varepsilon_{3} I & 0 & 0 \\ N_{C_{1}} X & 0 & 0 & 0 & 0 & 0 & -\varepsilon_{4} I & 0 \\ N_{D_{12}} Y & 0 & 0 & 0 & 0 & 0 & 0 & -\varepsilon_{5} I\end{array}\right]$ 
$\Pi=A X+X A^{\top}+B Y+Y^{\top} B^{\top}+\varepsilon_{1} M_{A} M_{A}^{\top}+\varepsilon_{2} M_{B} M_{B}^{\top}$

where

$\psi_{10}<0, P>0, X>0, \varepsilon_{1}>0, \varepsilon_{1}>0, \varepsilon_{1}>0, \varepsilon_{1}>0, \varepsilon_{1}>0$.

The gain matrix can be obtained as [31],

$K=Y X^{-1}$

So, finally, it can be rewritten as a Theorem:

Theorem 1 Consider the following system, including disturbance and uncertainty

$\left\{\begin{array}{l}\dot{x}=A(t) x+D(t) w+B(t) u \\ z=C_{1}(t) x+D_{11}(t) w+D_{12}(t) u \\ y=C_{2}(t) x+D_{21}(t) w+D_{22}(t) u\end{array}\right.$

where $\left\{\begin{array}{r}A(t)=A+\Delta A(t) \\ B(t)=B+\Delta B(t) \\ D(t)=D+\Delta D(t), \\ C_{1}(t)=C_{1}+\Delta C_{1}(t) \\ D_{12}(t)=D_{12}+\Delta D_{12}(t)\end{array} \quad \begin{array}{r}\Delta A(t)=M_{A} F(t) N_{A} \\ \Delta B(t)=M_{B} F(t) N_{B} \\ \Delta D(t)=M_{D} F(t) N_{D}, \\ \Delta C_{1}(t)=M_{C_{1}} F(t) N_{C_{1}} \\ \Delta D_{12}(t)=M_{D_{12}} F(t) N_{D_{12}}\end{array}\right.$ $\Delta A(t)=A_{1}(t) A_{2}(t) A_{3}(t), A_{1}(t)=M_{A} \in R^{n \times k} A_{3}(t)=N_{A} \in R^{l \times n}$, $A_{2}(t)=F(t) \in R^{k x l}, y=x, u=K x, \quad X=P^{-1}, Y=K P^{-1}$, $D_{11}=D_{21}=D_{22}=0$ and $C_{2}=l$.

For any $\psi_{10}<0, P>0, X>0, \varepsilon_{1}>0, \varepsilon_{1}>0, \varepsilon_{1}>0, \varepsilon_{1}>$ $0, \varepsilon_{1}>0$, by solving LMI $\psi_{10}$, the robust controller matrix with the ability of eliminating disturbances and uncertainties can be obtained as: $K=Y X^{-1}$

So, if the LMI $\psi_{10}<0$ is feasible for a system, where $\psi_{10}$ is defined by (45), due to the order of the equations and their mathematical proofs, the system will also be asymptotically stable, and the design is to be considered as robust.

Next, to model the uncertainties, first, the maximum and minimum of all the variable elements of the system state space matrices with uncertain parameter are calculated and then, their average value are obtained. Following on from the previous steps, the deviation of each element is calculated from its constant value, and finally, the proposed system is ready to be simulated by placing the constant elements (average part) in the matrices $A, B$ and $D_{\mathrm{w}}$ and also the variable elements in $\Delta A, \Delta B$ and $\Delta C$. The following equations give mathematical expression for the above steps.

$a_{i j \min } \leq a_{i j}(t) \leq a_{i j \max } b_{i j} \min \leq b_{i j}(t) \leq b_{i j \max ^{\prime}}$

$d_{w_{i j} \min } \leq d_{w_{i j}}(t) \leq d_{w_{i j} \max }$ $a_{i j \mathrm{~m}}=\frac{a_{i j \max }+a_{i j} \min }{2}, b_{i j_{\mathrm{m}}}=\frac{b_{i j \max }+b_{i j} \min }{2}$,

$d_{w_{i j \mathrm{~m}}}=\frac{d_{w_{i j} \max }+d_{w_{i j}} \min }{2}$

$h_{A_{i j}}=\frac{a_{i j \max }-a_{i j} \min }{2}, h_{B_{i j}}=\frac{b_{i j \max }-b_{i j} \min }{2}$,

$h_{D_{i j}}=\frac{d_{w_{i j} \max }-d_{w_{i j} \max }}{2}$

Therefore, for the matrix $A$,

$a_{11}=-R / L$

$a_{11 \text { max }}=\max \left(a_{11}\right), a_{11}$ min $=\min \left(a_{11}\right)$

$a_{11_{m}}=0.5\left(a_{11 \text { max }}+a_{11 \text { min }}\right)$

$h_{A_{11}}=0.5\left(a_{11 \text { max }}-a_{11}\right.$ min $)$

$a_{13}=-p \phi_{m} / L$

$a_{13 \text { max }}=\max \left(a_{13}\right), a_{13}$ min $=\min \left(a_{13}\right)$

$a_{13_{m}}=0.5\left(a_{13 \text { max }}+a_{13 \text { min }}\right)$

$h_{A_{13}}=0.5\left(a_{13 \max }-a_{13}\right.$ min $)$

$a_{31}=K_{t} / J$

$a_{31 \text { max }}=\max \left(a_{31}\right), a_{31}$ min $=\min \left(a_{31}\right)$

$a_{31_{m}}=0.5\left(a_{31}\right.$ max $+a_{31}$ min $)$

$h_{A_{31}}=0.5\left(a_{31} \max -a_{31}\right.$ min $)$

$a_{33}=-B / J$

$a_{33 \text { max }}=\max \left(a_{33}\right), a_{33}$ min $=\min \left(a_{33}\right)$

$a_{33_{m}}=0.5\left(a_{33 \text { max }}+a_{33}\right.$ min $)$

$h_{A_{33}}=0.5\left(a_{33 \max }-a_{33 \text { min }}\right)$

Thus, $A$ and $\Delta A$ are obtained as follows.

$A=\left[\begin{array}{cccc}a_{11_{m}} & 0 & a_{13_{m}} & 0 \\ 0 & 0 & 1 & 0 \\ a_{31_{m}} & 0 & a_{33_{m}} & 0 \\ 0 & -1 & 0 & 0\end{array}\right], \Delta A=\left[\begin{array}{cccc}h_{A_{11}} \beta & 0 & h_{A_{13}} \beta & 0 \\ 0 & 0 & 0 & 0 \\ h_{A_{31}} \beta & 0 & h_{A_{33}} \beta & 0 \\ 0 & 0 & 0 & 0\end{array}\right]$

where

$\beta=(-1+2(\mu))$

that $\mu$ is a random value between 0 and 1 . 
Similarly, the elements of matrices $B, \Delta B, D_{w}$ and $\Delta D_{w}$ are also calculated. Obviously, by knowing $\Delta A, \Delta B$ and $\Delta D_{w}$, from Eqs. (8), (9) and (10), $M_{A}, M_{B}, M_{D_{w}}$ and also $N_{A}, N_{B}$ and $N_{D_{w}}$ can be achieved.

So far, the design of the robust controller via uncertainty and disturbance elimination LMI has been defined. The next step is to improve the performance of the designed controller and develop it through gain-scheduling technique. Section 4 shows the simulation results of the controllers separately.

The classic gain-scheduling design process includes three steps. First, selecting several operating points that can cover a wide range of system dynamics, then at each operating point, linear approximation of time-invariant system can be obtained. Second, designing an LTI controller for each linearized system to meet operating conditions and finally, internalize the local linear design using the scheduling scheme to obtain a general nonlinear controller that can cover the entire operating ranges. By changing the operating conditions, feedback gains are recalculated. The idea of gain-scheduling technique has many applications and has acceptable performance over the entire operating ranges.

Thus, to implement the gain-scheduling controller, instead of using one robust controller for all operating points, several controllers can be used, and by measuring the speed feedbacks, the control system opt for the appropriate controller. To give more simplification, it can be said that, for example, if $1000 \leq \omega \leq 2000$, then the defined interval [1000 2000] is divided into some smaller intervals and for each interval a robust controller is designed. The main controller switches between sub-controllers as speed feedback changes during different conditions. This way, a more accurate control system is obtained. Section 4.3 is presented to show the performance of this type of controller.

\section{Simulation and results}

The parameters of the PMSM and system used in the simulations are given in Table 1. Moreover, the values of $L$ and $J$ are defined in terms of changes due to load variations and given specifications of the PMSM in abnormal conditions, are expressed as follows.

$L_{\min } \leq L \leq L_{\max }$

$J_{\min } \leq J \leq J_{\max }$

w h e r e $L_{\min }=0.005 \mathrm{H}, L_{\max }=0.015 \mathrm{H}$

a n d
Table 1 Specifications of PMSM

\begin{tabular}{lll}
\hline Parameters & Symbol & Value \\
\hline Stator resistance & $R$ & $0.54 \Omega$ \\
Pole pairs & $p$ & 4 \\
Viscous coefficient & $B$ & $0.0001 \mathrm{Nm} / \mathrm{rad} / \mathrm{s}$ \\
Magnetic flux & $\emptyset_{\mathrm{m}}$ & $0.61 \mathrm{wb}$ \\
Nominal current & $I_{\mathrm{n}}$ & $20 \mathrm{~A}$ \\
Nominal speed & $\omega_{\mathrm{n}}$ & $2000 \mathrm{rpm}$ \\
Nominal power & $P$ & $18 \mathrm{~kW}$ \\
Nominal torque & $T_{n}$ & $85 \mathrm{Nm}$ \\
\hline
\end{tabular}

After introducing the system and parameters as well as the robust controller design method, the simulation and results using YALMIP toolbox [35] are presented.

\subsection{Simulation, comparison, and results of robust and classic state feedback controllers}

In this section, six different scenarios are discussed as listed in Table 2. It has got merit to be mentioned that the state feedback controller presented in this section is designed with a simple pole-placement method. The system control scheme is shown in Fig. 1.

Table 2 Different scenarios of robust controller implementation in comparison with the classic state feedback controller

\begin{tabular}{lll}
\hline Scenario 1: without uncertainty & $T_{\mathrm{L}}=20 \mathrm{Nm}$ & $\gamma=0.2$ \\
Scenario 2: by \%100 uncertainty & $T_{\mathrm{L}}=20 \mathrm{Nm}$ & $\gamma=0.4$ \\
Scenario 3: without uncertainty & $T_{\mathrm{L}}=100 \mathrm{Nm}$ & $\gamma=0.2$ \\
Scenario 4: without uncertainty & $T_{\mathrm{L}}=20 \mathrm{Nm}$ & $\gamma=5$ \\
Scenario 5: by \%100 uncertainty & $T_{\mathrm{L}}=20 \mathrm{Nm}$ & $\gamma=5$ \\
Scenario 6: without uncertainty & $T_{\mathrm{L}}=100 \mathrm{Nm}$ & $\gamma=5$ \\
\hline
\end{tabular}

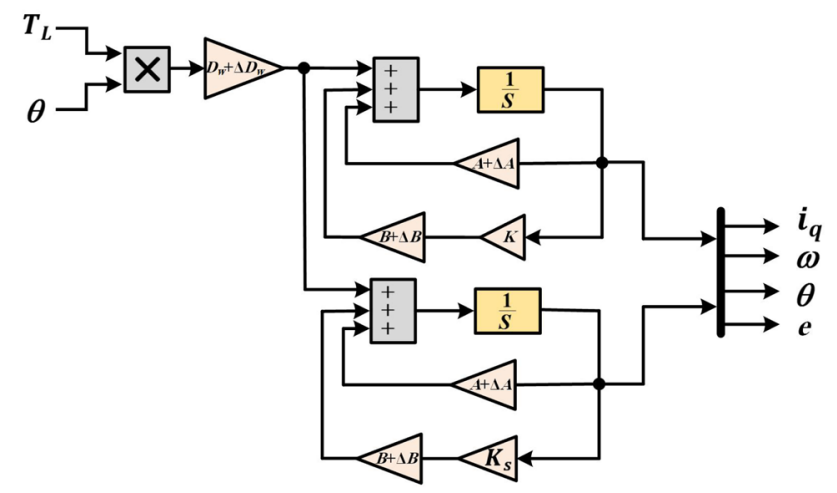

Fig. 1 Block diagram of control system (robust and classic state feedback controllers) 
As it has been presented in Fig. 2, two robust (upper part blocks) and classic state feedback (lower part blocks) controllers are observed. $K$ is the robust controller gain and $K_{\mathrm{S}}$ refers to the state feedback controller gain. $A+\Delta A, B+\Delta B$ and $D_{w}+\Delta D_{w}$ are obtained from (54) equation.

Scenario (1) The best value is opted for $\gamma$. Here, $\gamma$ is modified manually so that the LMI (45) would be feasible. Also, When the motor is running at a steady state, the load torque $T_{\mathrm{L}}=20 \mathrm{~N} \mathrm{~m}$ is applied suddenly to the motor at $t=20 \mathrm{~s}$. It is observed that the robust controller exhibits better performance in speed tracking under this condition. In this case where $\gamma=0.2$, the output currents of controllers do not exceed the system's nominal current. Although the LMI can be feasible for smaller values of $\gamma$, the limit of the output current may exceed nominal current and damage the system.
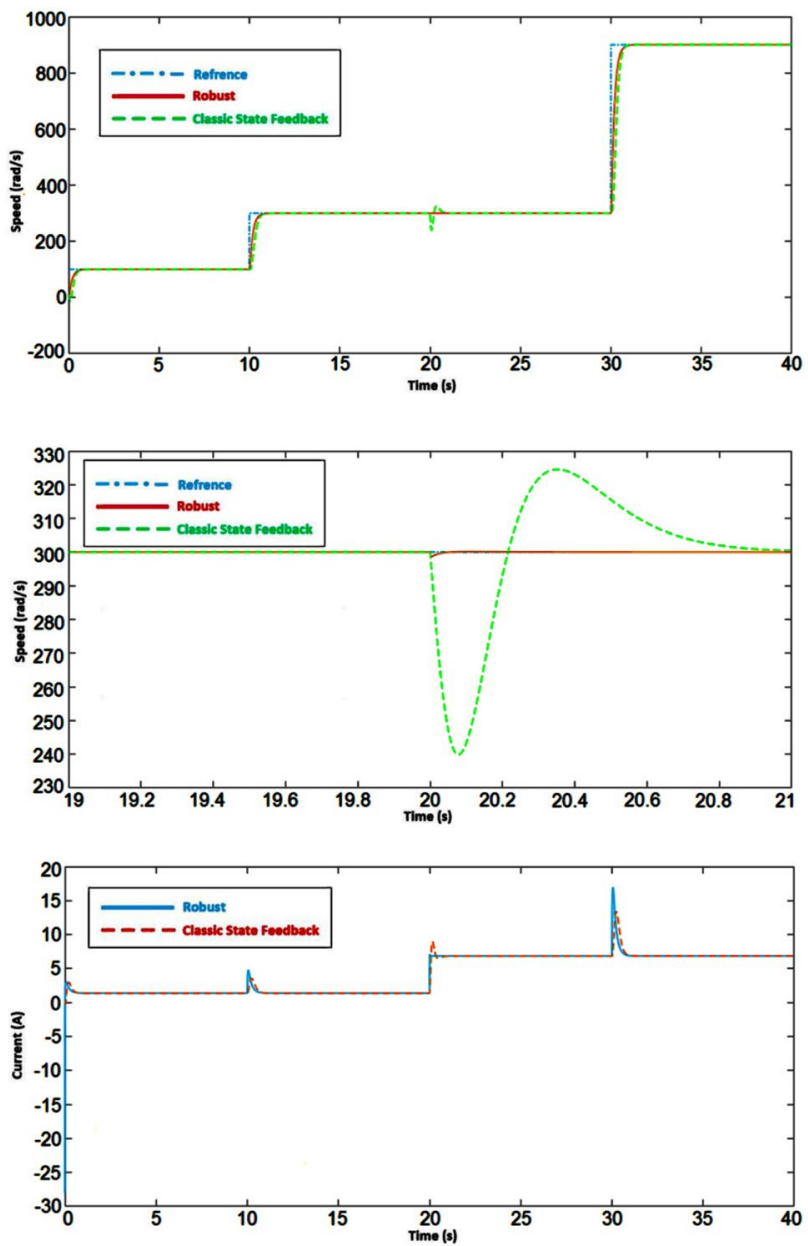

Fig. 2 Simulation results of scenario (1). a Speed tracking performance. b Accurate observation of speed tracking performance at $t=20$ s. c System output current
According to Fig. $2 b$, it is observed that the robust controller follows the reference speed immediately with a change of $1 \mathrm{rad} / \mathrm{s}$ after the disturbance, but the classic state feedback controller experiences speed changes of $60 \mathrm{rad} / \mathrm{s}$ and returns to its original state for a longer period of time.

Scenario 2 Optimal $\gamma$ is considered to be 0.4 in order to observe the current limit (Fig. 3).

Scenario 3 The torque applied to the system is increased to $100 \mathrm{~N} \mathrm{~m}$.

It can be seen from Fig. $4 \mathrm{~b}$ that the robust controller follows the reference speed immediately with a change of $2 \mathrm{rad} / \mathrm{s}$ after the disturbance, but the classic state feedback controller experiences speed changes of $136 \mathrm{rad} / \mathrm{s}$ and returns to its original state for a longer period of time.
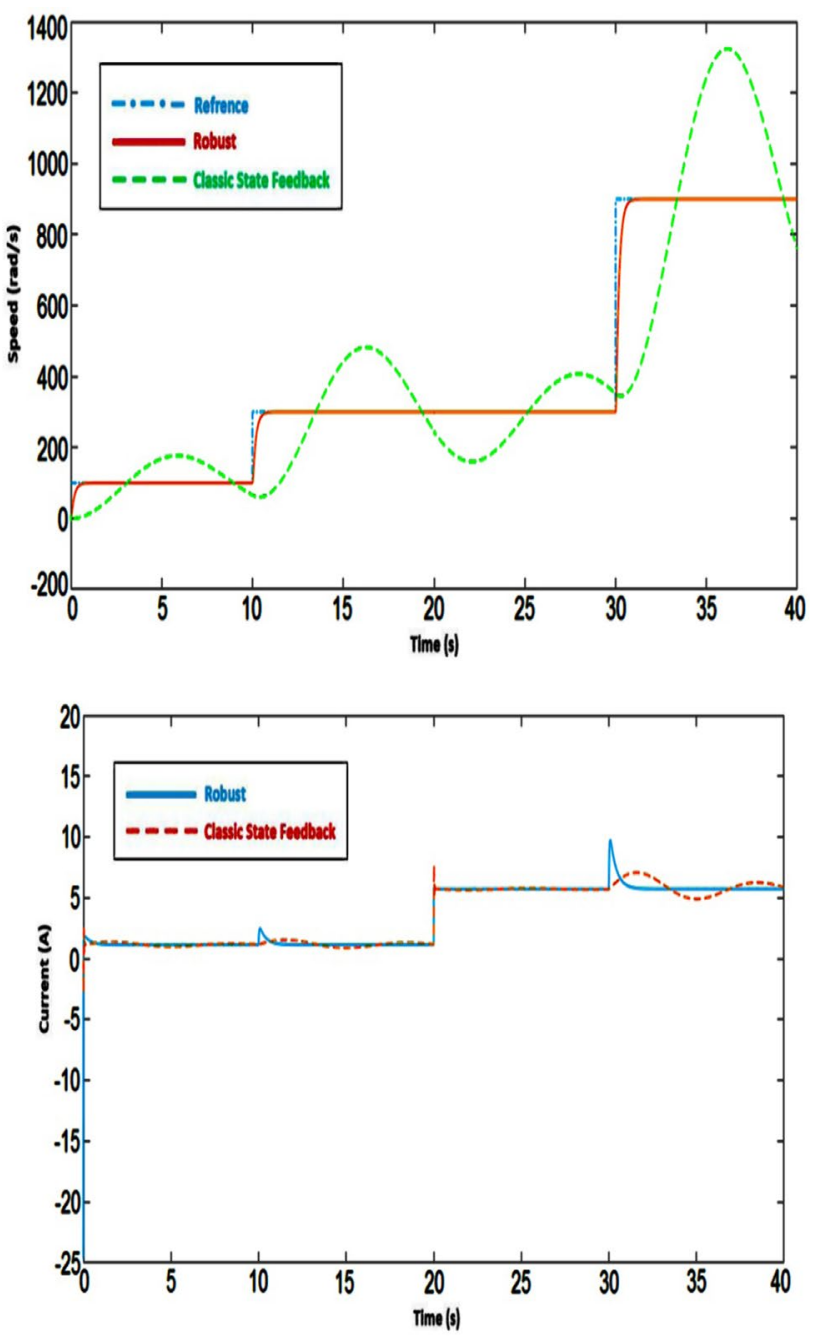

Fig. 3 Simulation results of scenario (2). a Speed tracking performance. b System output current 

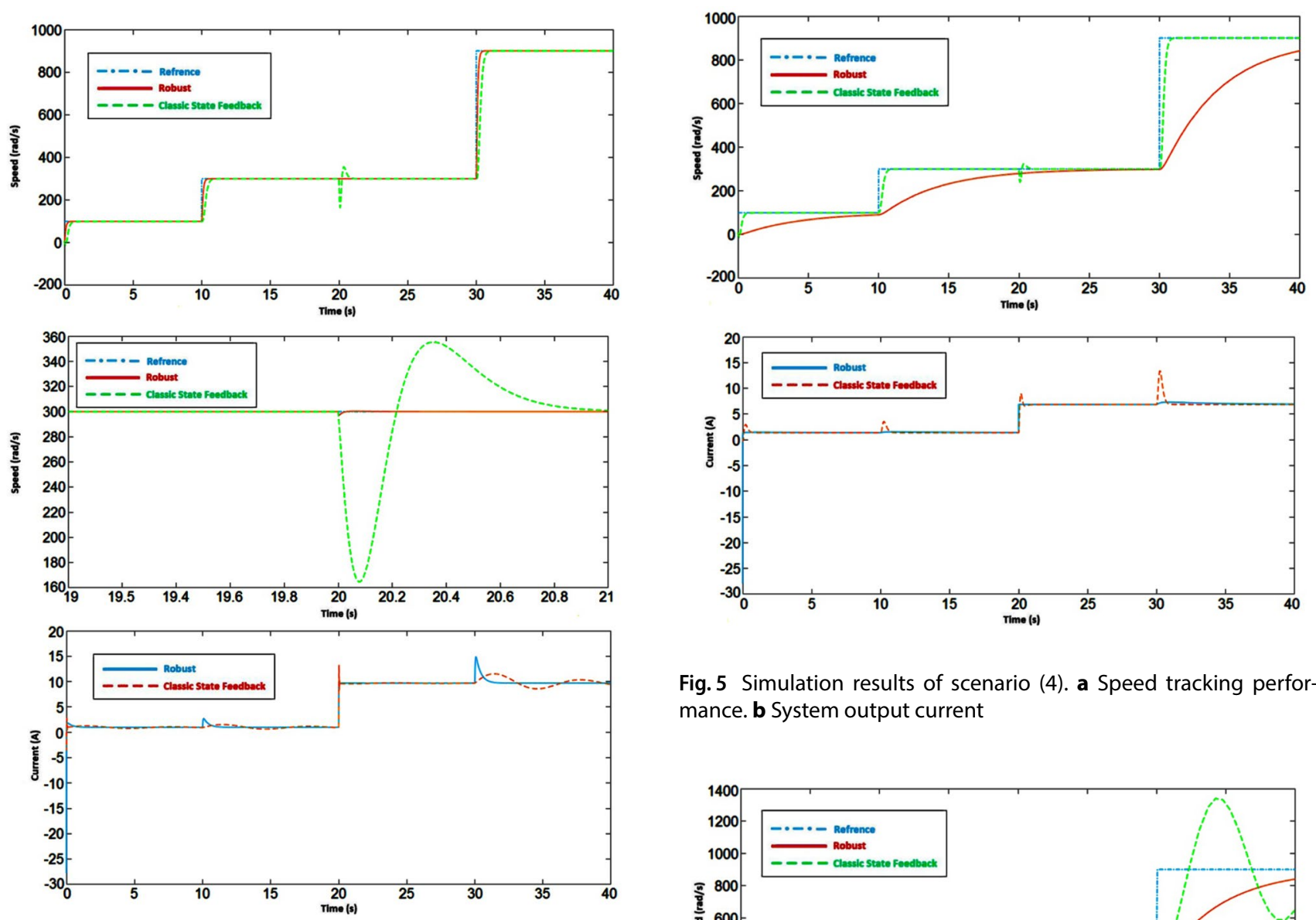

Fig. 4 Simulation results of scenario (3). a Speed tracking performance. $\mathbf{b}$ Accurate observation of speed tracking performance at $t=20$ s. c System output current

In order to observe the effect of $\gamma$ parameter on the performance of the robust controller, in the scenarios 4, 5, and $6, \gamma$ equal to 5 is introduced.

Scenario 4 Similar to scenario 1 with $\gamma$ equal to 5 (Fig. 5).

Scenario 5 Similar to scenario 2 with $\gamma$ equal to 5 (Fig. 6).

Scenario 6 Similar to scenario 3 with $\gamma$ equal to 5 (Fig. 7).

It can be observed that compared with the PI method, the PFC method shows a better disturbance rejection ability, with less speed fluctuations and shorter recovering times against disturbances.

Fig. 5 Simulation results of scenario (4). a Speed tracking performance. $\mathbf{b}$ System output current
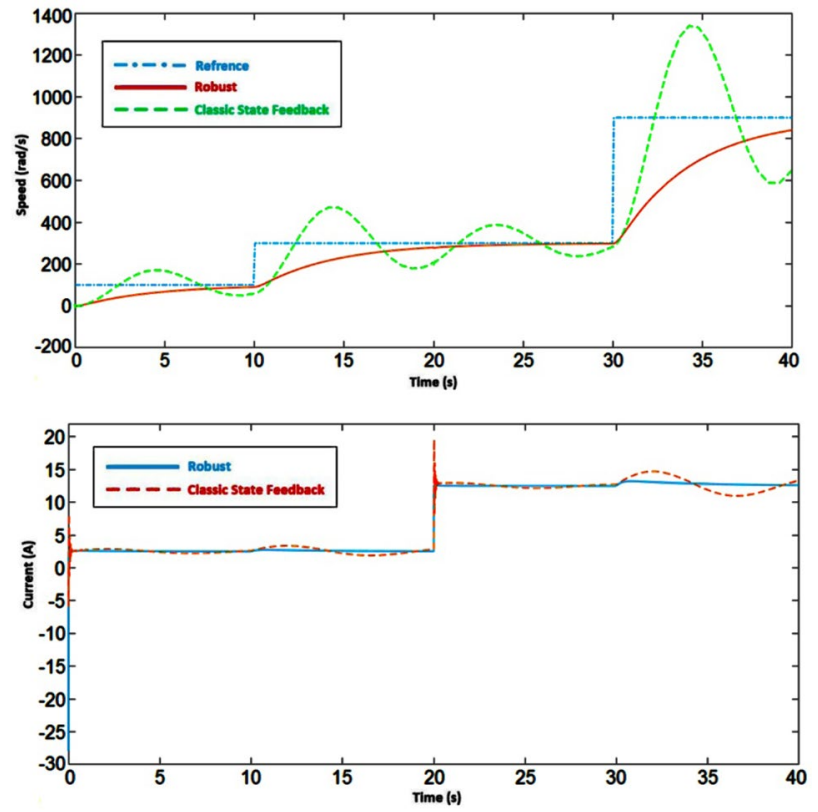

Fig. 6 Simulation results of scenario (5). a Speed tracking performance. b System output current 

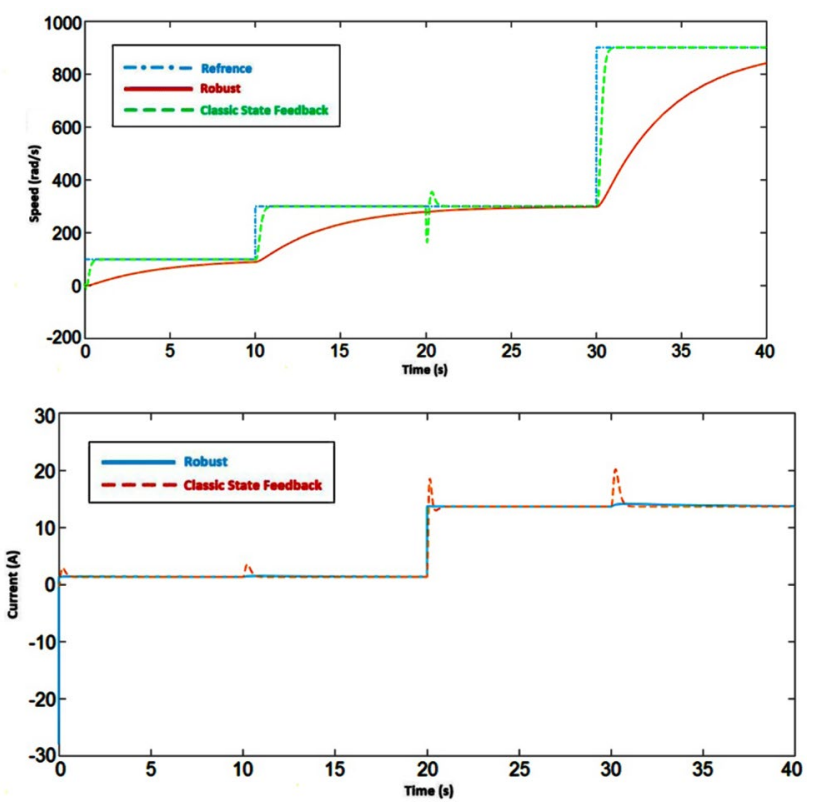

Fig. 7 Simulation results of scenario (6). a Speed tracking performance. $\mathbf{b}$ System output current

\subsection{Simulation, comparison and results of robust and PI controllers with parameters optimized by genetic algorithm (GA)}

The system block diagram with a PI controller is shown in Fig. 8. The optimized proportional and integral parameters of the controller are obtained by genetic algorithm.

In this simulation, the population is assumed to be 8 and the iteration is 20 . The output are $[K(1) K(2)]$ after 20 iterations, which equal to [1 63.2483]. The convergence diagram of the algorithm in this case can be seen in Fig. 9 .

Since the initial population is randomly selected [36], each time the algorithm is run, the gain values are

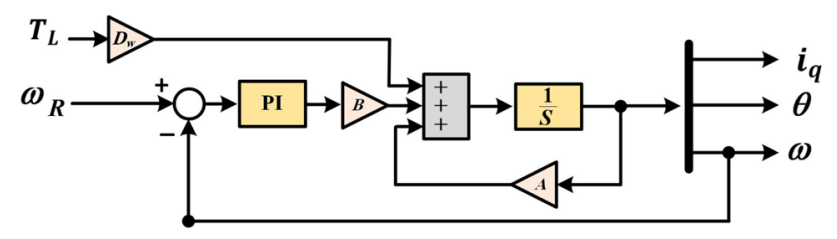

Fig. 8 System block diagram with PI controller

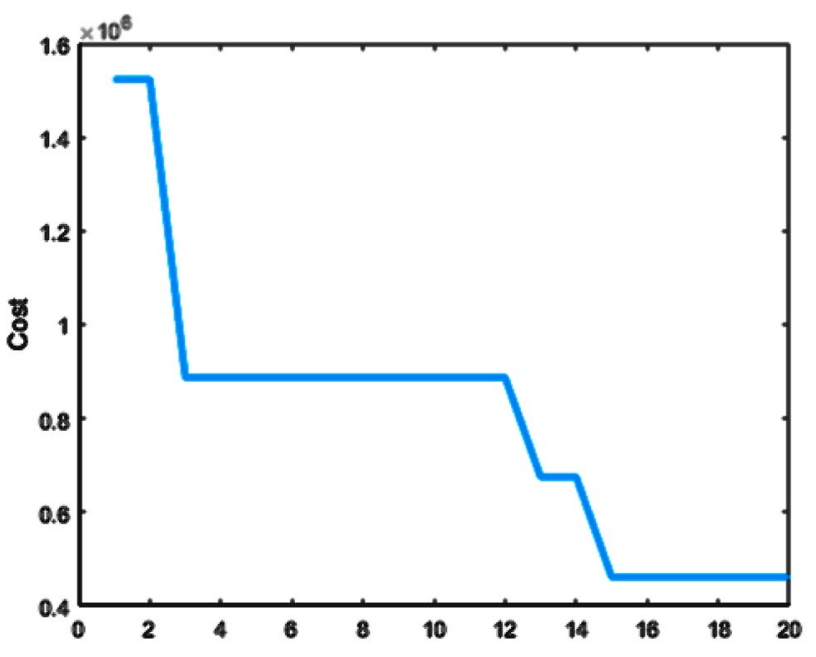

Fig. 9 Convergence diagram of the genetic algorithm

calculated and used as the optimized parameters. It can also be possible to change the initial population size and the number of iterations, but eventually the answers would be close to each other. Then, the optimized gains according to Fig. 10, are placed in the PI controller block and compared with the robust one.

$\gamma$ is set to 0.15 . The uncertainty is $100 \%$ applied to both control systems, and the torque applied at $t=20 \mathrm{~s}$ to the system in the form of disturbance equal to $20 \mathrm{~N} \mathrm{~m}$.

There are several conditions. The first is that for some uncertainties, the PI control system becomes unstable. In the latter case, for some uncertainties, the PI controller has a better performance in speed tracking, but it is observed by referring to the current diagrams that it has exceeded the current limit and this is not desirable. Of course, a small amount of $\gamma$ can be set for the robust controller that always performs better than the PI controller in speed tracking conditions, but the current limit would be exceeded. Therefore, the lowest $\gamma$ is selected to meet the current limit and the two control systems operate optimally. Figure 11 shows these cases.

Therefore, it can be seen that the robust controller has a much better performance. In the following, $\gamma$ is set to 0.06 to observe the effects of its reduction. As it has been shown in Fig. 12, it is true that lower $\gamma$ (such that robust controller LMI can be feasible) has a better speed tracking 


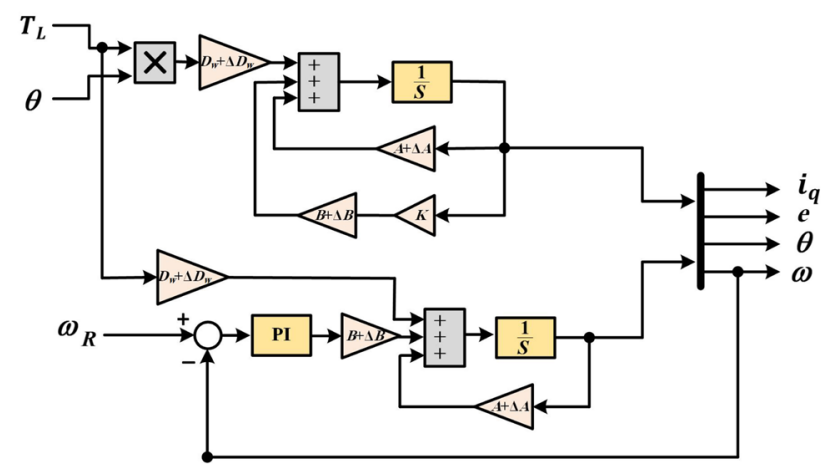

Fig. 10 Block diagram of control system (robust and PI controllers) than the PI controller (which parameters are optimized by the genetic algorithm) but does not meet the current limit. Therefore, as explained above, it is significant to look for the lowest $\gamma$ so that the output current of the system for the robust control system does not exceed the system current limit.

By using the robust controller, the system is stable for all values of uncertainties and applied disturbances. Thus, it can be said that the reliability of this type of controller is higher than the others (state feedback and PI controllers).
Fig. 11 Simulation results of robust controller compared with PI controller (parameters optimized by genetic algorithm). a Control system instability by $\% 100$ uncertainty. b Speed tracking performance by less than $\% 50$ uncertainty. c Accurate observation of speed tracking performance at $t=30 \mathrm{~s}$. d System output current. e Accurate observation of system output current at $t=10 \mathrm{~s}$
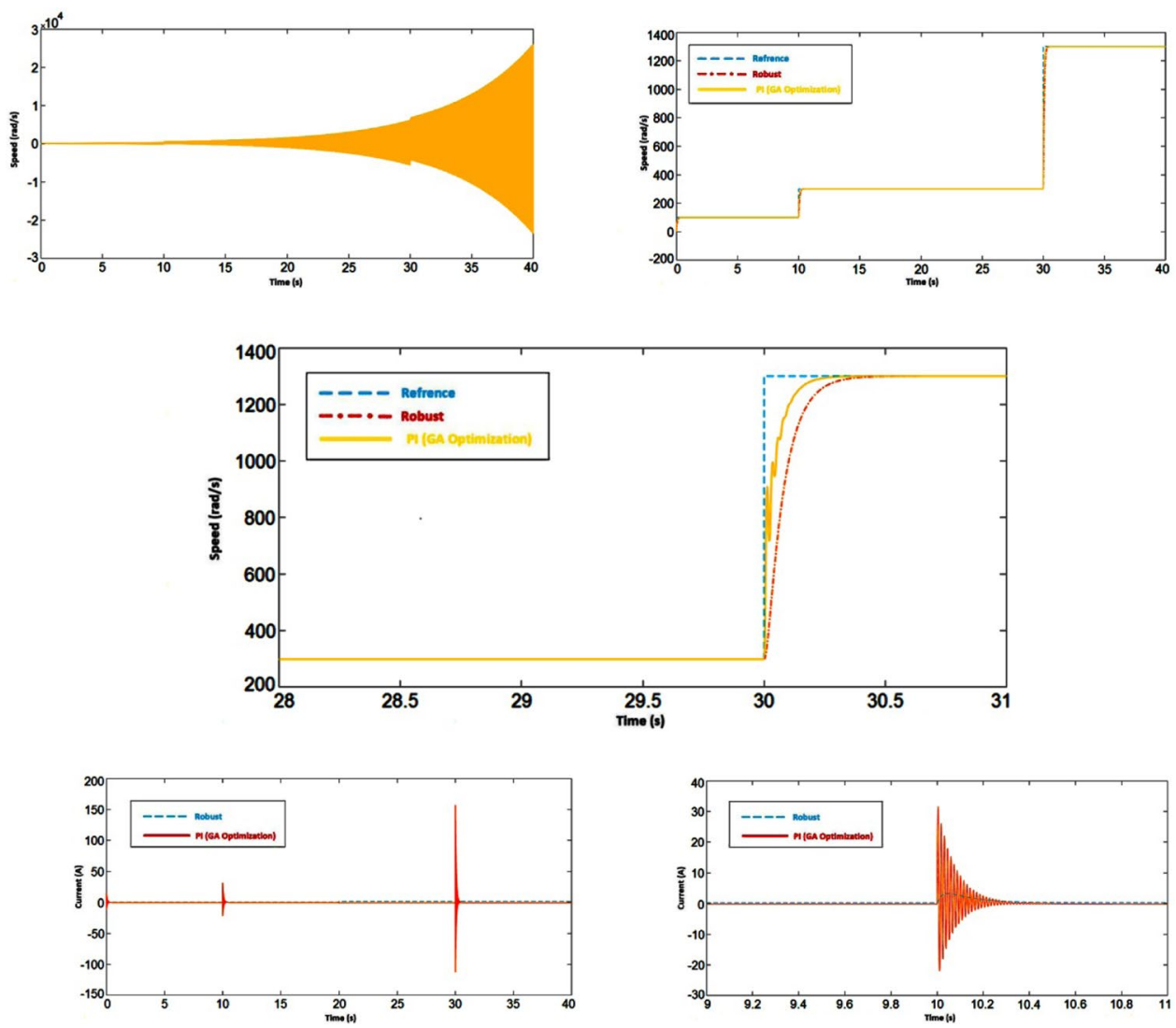

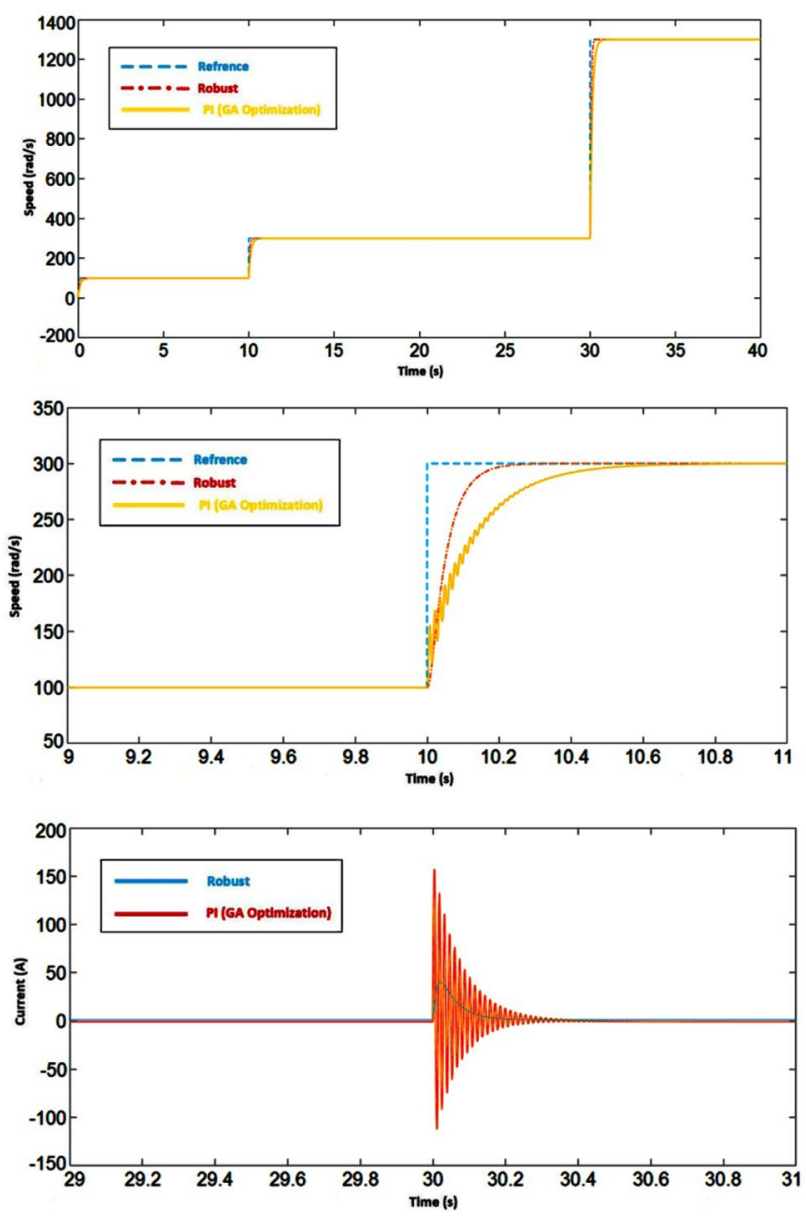

Fig. 12 Effects of $\gamma$ reduction on speed tracking performance and output current. a Speed tracking performance. b Accurate observation of speed tracking performance at $t=10 \mathrm{~s}$. c Accurate observation of system output current at $t=10 \mathrm{~s}$

\subsection{Simulation and results of gain-scheduled control method}

The system under study in this part is the one discussed in Eq. (6) except that the reference current on the axis $d$ is not considered zero and the new system equations are defined as follows.

$A=\left[\begin{array}{ccccc}-\frac{R}{L} & -p \omega & \frac{p \phi_{\mathrm{m}}}{L} & 0 & 0 \\ p \omega & -\frac{R}{L} & 0 & 0 & 0 \\ \frac{K_{\mathrm{t}}}{J} & 0 & -\frac{B}{J} & 0 & 0 \\ 0 & 0 & 1 & 0 & 0 \\ 0 & 0 & 0 & -1 & 0\end{array}\right] B=\left[\begin{array}{cc}\frac{1}{L} & 0 \\ 0 & \frac{1}{L} \\ 0 & 0 \\ 0 & 0 \\ 0 & 0\end{array}\right] D_{w}=\left[\begin{array}{cc}0 & 0 \\ 0 & 0 \\ -\frac{1}{J} & 0 \\ 0 & 0 \\ 0 & 1\end{array}\right] C_{\mathrm{Z}}=\left[\begin{array}{lllll}0 & 0 & 0 & 0 & 1\end{array}\right]$,

w h $\quad$ e $\quad r \quad$ e $\quad x(t)=\left[i_{q}(t) i_{d}(t) \omega(t) \theta(t) e(t)\right]^{\top}$, $u(t)=\left[u_{q}(t) u_{d}(t)\right]^{\top}$ is the control input and $\mathrm{d}(t)=\left[\begin{array}{ll}\theta^{*} & T_{\mathrm{L}}\end{array}\right]^{\top}$ refers to disturbance input.
Here, $\gamma$ equal to 0.1 and he controller gain is calculated for several speed intervals (operating points). At $t=20 \mathrm{~s}$, $T_{\mathrm{L}}=50 \mathrm{~N} \mathrm{~m}$ is applied to the motor.

Due to the wide variation in the dynamic properties of electrical systems, gain-scheduled control method is an appropriate design strategy to enhance the robustness of the system. The work done here is to get feedback from speed and compare it with the reference speed of the proposed system. In fact, instead of using one controller for all operating points, several controllers are used depending on the speed at which the PMSM is running. That is, for each speed interval, different gains are utilized. The controllers designed for each part are named from $K_{1}$ to $K_{n}$, $n=1, \ldots, \infty$. If $n$ equal to $\infty$, it means that there is a robust controller for every operating point and it demonstrates the pure meaning of a gain-scheduled control method.

Obviously, in the moments of reference speed change, there are severe disturbances in the system that occur over a short period of time. The reason is that with the sudden change of speed, the controller gain also switches abruptly and with a large change amplitude. For example, a gain fluctuates from 100 to -600 within a short period of time, causing enormous perturbations.

Under real conditions, due to the high inertia of the PMSM, the speed cannot be changed abruptly in a short period of time, but the gain $K$ is slowly changed to observe results with less fluctuation. So that from $K_{1}$ to $K_{2}$, the gain changes gently. In fact, this is done by creating a time delay between the different intervals. For example, for $\omega \leq 200 \mathrm{rad} / \mathrm{s}, K=K_{1}$. Between the 10th to the 13th seconds which $200<\omega \leq 400 \mathrm{rad} / \mathrm{s}$ the shift function $K$ can be defined as follows.

$K=K_{2}+\left(K_{1}-K_{2}\right) *((13-t) / 3)^{2}$

Between the 30th to 38th seconds which $400<\omega \leq 600$ the shift function $K$ can be presented as

$K=K_{3}+\left(K_{2}-K_{3}\right) *((38-t) / 8)^{1.8}$

The system block diagram for gain-scheduled controller can be seen in Fig. 13.

The Function block is made up of speed intervals and Eqs. (58) and (59).

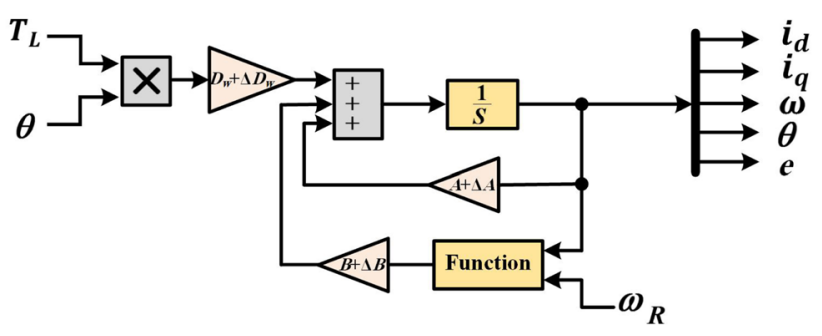

Fig. 13 Block diagram of proposed gain-scheduled control system 


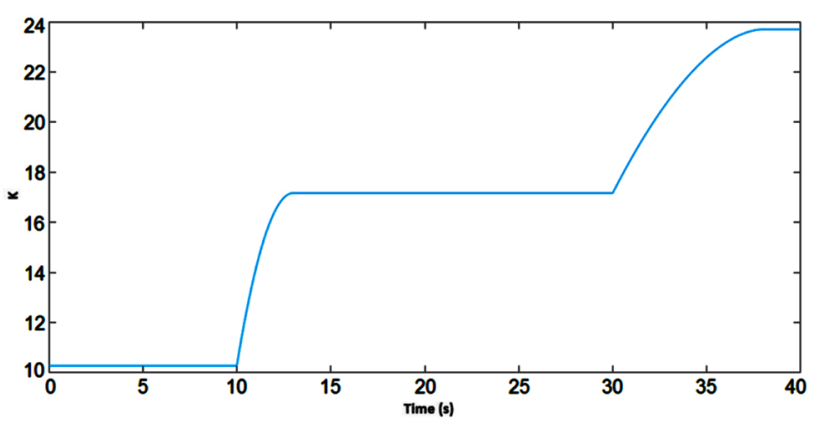

Fig. 14 Gain diagram with softer changes
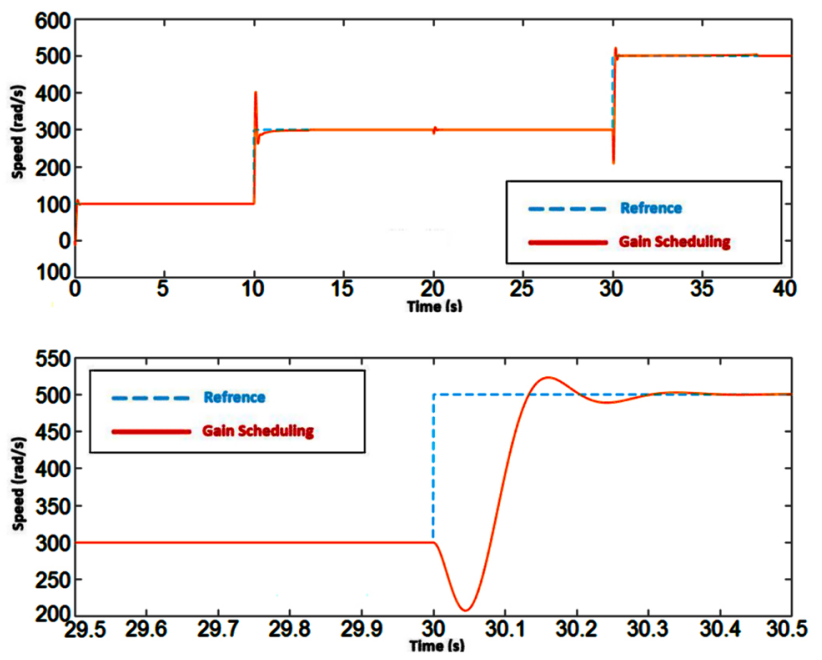

Fig. 15 Simulation results of gain-scheduled control method. a Speed tracking performance. b Accurate observation of speed tracking performance at $t=30 \mathrm{~s}$

Thus, in this way, as it can be observed from Fig. 14, gain changes from one value to another smoothly.

The simulation results are shown in Fig. 15.

In general, it can be stated that designing a controller for all operating points and uncertainty intervals is less accurate than a gain-scheduled controller, and it must be noticed that Controllers designed for different operating conditions act more accurate than the others.

\section{Conclusion}

In this paper, a robust gain-scheduled control method via LMI approach has been adopted for PMSM speed control. By using control theories and also uncertainty and disturbance elimination LMI which was obtained step by step, robust controller was designed. Classic state feedback and $\mathrm{PI}$ controllers were also designed to compare their performances with robust controller. An effective genetic algorithm was implemented on PI parameters respectively. Then, by applying the gain-scheduled technique, several robust controllers were designed for different operating points. Proposed control scheme guarantees the robustness under the existence of parameter uncertainties and disturbances. It is worth noting that this speed control system can be applied to high-performance applications in different industries.

Author contributions All authors contributed to the study conception and design. Material preparation, data collection and analysis were performed by MHM, MA, and MEK. The first draft of the manuscript was written by MHM, PS, and FV and of course all authors commented on previous versions of the manuscript. All authors read and approved the final manuscript.

\section{Compliance with ethical standards}

Conflict of interest The authors declare that they have no conflict of interest.

\section{References}

1. Türker T, Buyukkeles U, Bakan AF (2016) A robust predictive current controller for PMSM drives. IEEE Trans Ind Electron 63:39063914. https://doi.org/10.1109/TIE.2016.2521338

2. Yang M, Lang X, Long J, Xu D (2017) Flux immunity robust predictive current control with incremental model and extended state observer for PMSM drive. IEEE Trans Power Electron 32:9267-9279. https://doi.org/10.1109/TPEL.2017.2654540

3. Caii R, Zheng R, Liu M, Li M (2017) Robust control of PMSM using geometric model reduction and $\mu$ synthesis. IEEE Trans Ind Electron 65:498-509. https://doi.org/10.1109/TIE.2017.2714140

4. Liu X, Zhang Q (2019) Robust current predictive control-based equivalent input disturbance approach for PMSM drive. Electronics 8:1034. https://doi.org/10.3390/electronics8091034

5. Hassaine S, Moreau S, Ogab C, Mazari B (2007) Robust speed control of PMSM using generalized predictive and direct torque control techniques. IEEE Int Symp Ind Electron. https://doi. org/10.1109/ISIE.2007.4374771

6. Zhang X, Sun L, Zhao K, Sun L (2012) Nonlinear speed control for PMSM system using sliding-mode control and disturbance compensation techniques. IEEE Trans Power Electron 28:1358-1365. https://doi.org/10.1109/TPEL.2012.2206610

7. Do TD, Choi HH, Jung JW (2014) $\Theta-D$ approximation technique for nonlinear optimal speed control design of surface-mounted PMSM drives. IEEE/ASME Trans Mechatron 20:1822-1831. https ://doi.org/10.1109/TMECH.2014.2356138

8. Rizvi SAA, Memon AY (2019) An extended observer-based robust nonlinear speed sensorless controller for a PMSM. Int J Control 92:2123-2135. https://doi.org/10.1080/00207 179.2018 .1428768

9. Li S, Liu Z (2009) Adaptive speed control for permanent-magnet synchronous motor system with variations of load inertia. IEEE Trans Ind Electron 56:3050-3059. https://doi.org/10.1109/ TIE.2009.2024655

10. Demenko A, Hameyer K, Pietrowski W, Šušnjić L, Zawirski K, Pajchrowski T (2013) Application of artificial neural network 
for adaptive speed control of PMSM drive with variable parameters. COMPEL Int J Comput Math Electr Electron Eng. https:// doi.org/10.1108/03321641311317103

11. Brock S, Deskur J, Zawirski K (1999) Robust speed and position control of PMSM. In: ISIE'99. Proceedings of the IEEE international symposium on industrial electronics, vol 2, pp 667-672. https://doi.org/10.1109/ISIE.1999.798692

12. Kim SK, Lee JS, Lee KB (2018) Robust speed control algorithm with disturbance observer for uncertain PMSM. Int J Electron 105:1300-1318. https://doi.org/10.1080/00207217.2018.14404 30

13. Li S, Zhou M, Yu X (2012) Design and implementation of terminal sliding mode control method for PMSM speed regulation system. IEEE Trans Ind Inf 9:1879-1891. https://doi.org/10.1109/ TII.2012.2226896

14. Liu X, Yu H, Yu J, Zhao L (2018) Combined speed and current terminal sliding mode control with nonlinear disturbance observer for PMSM drive. IEEE Access 6:29594-29601. https:// doi.org/10.1109/ACCESS.2018.2840521

15. Zaihidee FM, Mekhilef S, Mubin M (2019) Application of fractional order sliding mode control for speed control of permanent magnet synchronous motor. IEEE Access 7:101765-101774. https://doi.org/10.1109/ACCESS.2019.2931324

16. Elmas C, Ustun O, Sayan HH (2008) A neuro-fuzzy controller for speed control of a permanent magnet synchronous motor drive. Expert Syst Appl 34:657-664. https://doi.org/10.1016/j. eswa.2006.10.002

17. Sakunthala S, Kiranmayi R, Mandadi PN (2017) A study on fuzzy controller and neuro-fuzzy controller for speed control of PMSM motor. In: IEEE international conference on power, control, signals and instrumentation engineering (ICPCSI), pp 1409-1413. https://doi.org/10.1109/ICPCSI.2017.8391943

18. Bouguenna IF, Azaiz A, Tahour A, Larbaoui A (2019) Robust neuro-fuzzy sliding mode control with extended state observer for an electric drive system. Energy 169:1054-1063. https://doi. org/10.1016/j.energy.2018.12.101

19. Liu X, Zhang C, Li K, Zhang Q (2017) Robust current controlbased generalized predictive control with sliding mode disturbance compensation for PMSM drives. ISA Trans 71:542-552. https://doi.org/10.1016/j.isatra.2017.08.015

20. Shao M, Deng Y, Li H, Liu J, Fei Q (2019) Robust speed control for permanent magnet synchronous motors using a generalized predictive controller with a high-order terminal slidingmode observer. IEEE Access 7:121540-121551. https://doi. org/10.1109/ACCESS.2019.2937535

21. Bhattacharyya SP, Keel LH (1995) Robust control: the parametric approach. Adv Control Educ. https://doi.org/10.1016/B978-0-08042230-5.50016-5

22. Wang Y, Xie L, De Souza CE (1992) Robust control of a class of uncertain nonlinear systems. Syst Control Lett 19:139-149. https ://doi.org/10.1016/0167-6911(92)90097-C
23. Jan RM, Tseng CS, Liu RJ (2008) Robust PID control design for permanent magnet synchronous motor: a genetic approach. Electr Power Syst Res 78:1161-1168. https://doi.org/10.1016/j. epsr.2007.09.011

24. Kumar V, Gaur P, Mittal AP (2014) ANN based self-tuned PID like adaptive controller design for high performance PMSM position control. Expert Syst Appl 41:7995-8002. https://doi. org/10.1016/j.eswa.2014.06.040

25. Song Q, Jia C (2016) Robust speed controller design for permanent magnet synchronous motor drives based on sliding mode control. Energy Procedia 88:867-873. https://doi.org/10.1016/j. egypro.2016.06.102

26. Mendoza-Mondragón F, Hernández-Guzmán VM, RodríguezReséndiz J (2018) Robust speed control of permanent magnet synchronous motors using two-degrees-of-freedom control. IEEE Trans Ind Electron 65:6099-6108. https://doi.org/10.1109/ TIE.2017.2786203

27. Jon R, Wang Z, Luo C, Jong M (2017) Adaptive robust speed control based on recurrent Elman neural network for sensorless PMSM servo drives. Neurocomputing 227:131-141. https ://doi.org/10.1016/j.neucom.2016.09.095

28. Liu H, Li S (2011) Speed control for PMSM servo system using predictive functional control and extended state observer. IEEE Trans Ind Electron 59:1171-1183. https://doi.org/10.1109/ TIE.2011.2162217

29. Krishnan R (2001) Electric motor drives: modeling, analysis and control. Prentice Hall, Upper Saddle River

30. Sun X, Yi Y, Cao S, Liu H, Zhang T (2015) Robust PI-type position controller design for permanent magnet synchronous motor using LMI techniques. Lecture Notes Electr Eng 337:405-412. https://doi.org/10.1007/978-3-662-46463-2_41

31. Liu Y, Wang Z, Liu X (2007) Robust $\mathrm{H} \infty$ control for a class of nonlinear stochastic systems with mixed time delay. Int J Robust Nonlinear Control IFAC-Affil J 17(16):1525-1551. https://doi. org/10.1002/rnc. 1185

32. El Ghaoui L, Niculescu SI (eds) (2000) Advances in linear matrix inequality methods in control, vol 2. SIAM

33. Doyle JC, Francis BA, Tannenbaum AR (1990) Feedback control theory. Macmillan, New York

34. Zhang F (2006) The Schur complement and its applications. Springer, Berlin

35. Lofberg J (2004) YALMIP: a toolbox for modeling and optimization in MATLAB. In: Proceedings of the CACSD conference

36. Davis $L$ (1991) Handbook of genetic algorithms. Van Nostrand Reinhold, New York

Publisher's Note Springer Nature remains neutral with regard to jurisdictional claims in published maps and institutional affiliations. 